\title{
COHERENT SHEAVES ON FORMAL COMPLETE INTERSECTIONS VIA DG LIE ALGEBRAS
}

\author{
SAM RASKIN
}

\begin{abstract}
We establish a criterion for sheaves on an adically complete DG scheme to be coherent. We deduce a description of coherent sheaves on an adically complete lci singularity in terms of modules for a DG Lie algebra.
\end{abstract}

\section{CONTEnts}

1. Introduction 207

2. Coherent sheaves 211

3. The quasi-smooth case 215

Acknowledgments 218

$\begin{array}{ll}\text { Appendix A. Hessian calculations } & 218\end{array}$

$\begin{array}{ll}\text { References } & 222\end{array}$

\section{Introduction}

1.1. The aim for this note is to prove a coherence criterion for sheaves on an adically complete scheme.

We fix a ground field $k$ of characteristic zero for the remainder of this note.

1.2. Let us explain what the result says in the case of a complete intersection. Note that in Section 1.4 we will begin using the language of derived algebraic geometry; in Sections 1.2 and 1.3, the reader may understand the language used in the sense of either classical or derived algebraic geometry without any essential change to its meaning.

Let $Z$ be a complete intersection over $k$ given as a fiber product:

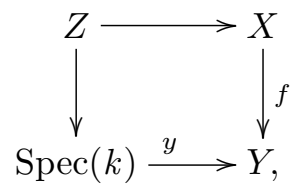

where $f$ is flat and $X$ and $Y$ are smooth schemes over $k$. Let $z \in Z$ be a closed point and let $\widehat{Z} \in$ Sch be the adic completion of $Z$ along $z$.

Let $\operatorname{Coh}(\widehat{Z})$ denote the DG category of bounded complexes of coherent sheaves on $\widehat{Z}$. Here the homotopy category of $\operatorname{Coh}(\widehat{Z})$ is the triangulated category $D^{b}\left(\operatorname{Coh}(\widehat{Z})^{\mathcal{}}\right)$,

Received by the editors March 2, 2012. 
the bounded derived category of finitely generated $\mathcal{O}_{\widehat{Z}}$-modules. We will give an alternative description of this DG category.

1.3. Recall that for $Z$ any scheme (over $k$ ) and $i_{z}: z \hookrightarrow Z$ a closed point, the shifted derived fiber $L i_{z}^{*}\left({ }^{R} T_{Z}[-1]\right)$ of the tangent complex ${ }^{R} T_{Z}$ admits a canonical DG Lie algebra (aliases: $L_{\infty}$-algebra, homotopy Lie algebra) structure. This structure is due essentially to $[10,11]$; see also the more modern references $[1,15]$.

In the case of a complete intersection as above, the complex $\mathfrak{g}_{Z, z}$ can be computed explicitly: it is just the two step complex in degrees 1 and 2 :

$$
T_{X, z} \rightarrow f^{*}\left(T_{Y, y}\right)
$$

where the differential is the derivative of $f$. While it is not so clear how to write a formula for the Lie bracket at the chain level in these terms, one can show that at the level of cohomology the Lie bracket is the classical Hessian map:

$$
\operatorname{Hess}(f): \operatorname{Sym}^{2}\left(\operatorname{Ker}\left(T_{X, z} \rightarrow f^{*}\left(T_{Y, y}\right)\right)\right) \rightarrow \operatorname{Coker}\left(T_{X, z} \rightarrow f^{*}\left(T_{Y, y}\right)\right) .
$$

We refer to the appendix for this computation.

Let $\mathfrak{g}_{Z, z}$-mod denote the DG category of modules for $\mathfrak{g}_{Z, z}$. Given a complex $M^{\bullet}$ with the structure of $\mathfrak{g}_{Z, z}$-module, we can take its cohomology $H^{*}(M)$ to obtain a graded module for the graded Lie algebra $H^{*}\left(\mathfrak{g}_{Z, z}\right)=H^{1}\left(\mathfrak{g}_{Z, z}\right) \oplus H^{2}\left(\mathfrak{g}_{Z, z}\right)$. Let $\mathfrak{g}_{Z, z}-\bmod _{f . g}$. denote the full DG subcategory of $\mathfrak{g}_{Z, z}-$ mod where we ask that $H^{*}(M)$ be finitely generated over $H^{*}\left(\mathfrak{g}_{Z, z}\right)$ (equivalently: as a $\operatorname{Sym}\left(H^{2}\left(\mathfrak{g}_{Z, z}\right)\right)$-module).

Theorem 1.1. Coh $(\widehat{Z})$ and $\mathfrak{g}_{Z, z}-\bmod _{f . g}$. are canonically equivalent DG categories.

Remark 1.2. Of course, this statement of the theorem is completely coordinate-free: its formulation makes no reference to $X$ or $Y$.

1.4. For the remainder of this text, We will treat derived algebraic geometry using the formalism of higher category theory as developed by Lure in $[13,17]$.

We find it particularly convenient to use the language and notation from [6] and we will follow loc. cit. in some abuses. In particular, "scheme" will always mean "derived scheme," "category" will always mean " $\infty$-category," (alias: $(\infty, 1)$-category) "DG category" will mean "stable $\infty$-category enriched over (complexes of) $k$-vector spaces," "QCoh $(X)$ " will denote the DG category of quasi-coherent sheaves on $X$ and so on. We use the term "commutative algebra" for a commutative algebra in Vect : $=\mathrm{Q} \operatorname{Coh}(\operatorname{Spec}(k))$ and "classical commutative algebra" for a commutative algebra in Vect $^{\Upsilon}$. We use the phrase "classical scheme" for a scheme in the classical sense, i.e., a derived scheme which is locally the spectrum of a classical commutative algebra. We let $X^{\mathrm{cl}}$ denote the classical scheme underlying a (derived) scheme $X$.

If $\mathcal{C}$ is a symmetric monoidal DG category, then by a "Lie algebra in $\mathcal{C}$ " we understand an algebra in $\mathcal{C}$ for the Lie operad. By a "Lie algebra," we understand a Lie algebra in Vect. The terms "algebra" and "commutative algebra" are similarly understood in this (operadic) sense.

Functors between DG categories will always commute with finite colimits. By a continuous functor, we understand a functor which commutes with all colimits. For $\mathcal{C}$ an $\infty$-category, we let Ind $(\mathcal{C})$ denote its ind-completion, i.e., the $\infty$-category obtained by freely adding all filtered colimits. 
In addition, we impose the requirement that "scheme" mean "separated, quasicompact (derived) scheme over $k . "$

We will also change notation from Sections 1.2 and 1.3 in the following mild way: all constructions will be assumed to be derived, so for example, we will write $i^{*}$ where we wrote $L i^{*}$, and we will denote the tangent complex of $X$ by $T_{X}$ rather than ${ }^{R} T_{X}$. Note that the tangent complex $T_{X} \in \mathrm{Q} \operatorname{Coh}(X)$ is defined for any derived scheme and $T_{X}[-1]$ is a Lie algebra in $\mathrm{Q} \operatorname{Coh}(X)$.

For $\mathcal{C}$ an $\infty$-category and $X, Y \in \mathcal{C}$, we let $\operatorname{Hom}_{\mathcal{C}}(X, Y)$ denote the groupoid (aliases: homotopy type, $\infty$-groupoid) of maps in $\mathcal{C}$.

Remark 1.3. One immediate advantage of derived schemes is that, with the notation above, we may omit the restriction that $f: X \rightarrow Y$ be flat at the expense that the fiber product $Z:=X \times_{Y} \operatorname{Spec}(k)$ is a derived scheme.

However, note that in this case $\operatorname{Coh}(\widehat{Z})$ will no longer be the bounded derived category of its heart.

1.5. Let us recall a bit of terminology which is somewhat non-standard.

If $\mathcal{C}$ is a DG category with $t$-structure, we say an object $M \in \mathcal{C}$ is connective if $M \in \mathcal{C}^{\leq 0}$ and coconnective if $M \in \mathcal{C}^{\geq 0}$ (here and everywhere we use the cohomological indexing). We say $M$ is eventually connective (alias: bounded above) if $M \in \mathrm{C}^{-}$and eventually coconnective (alias: bounded below) if $M \in \mathrm{C}^{+}$. For such $\mathcal{C}$, we let $\mathcal{C}^{\odot}$ denote the heart of the $t$-structure. For example, QCoh $(X)^{\odot}$ denotes the abelian category of quasi-coherent sheaves on $X$ (which we recall coincides with $\left.\mathrm{Q} \operatorname{Coh}\left(X^{\mathrm{cl}}\right)^{\ominus}\right)$.

We let AffSch denote the category of (derived) affine schemes, i.e., the opposite category to the category of commutative $k$-algebras which are connective as vector spaces. Let PreStk denote the category of prestacks, i.e., functors AffSch ${ }^{o p} \rightarrow \mathrm{Gpd}$, where Gpd is the category of $\infty$-groupoids. The category of (derived) schemes is by definition a full subcategory Sch $\subset$ PreStk.

Let $Y$ be a closed subscheme of a scheme $X$. Recall from [7] that the formal completion $X_{Y}^{\wedge}$ is the prestack whose $S$-points are the groupoid of maps $S \rightarrow X$ such that $S \times_{X}(X \backslash Y)=\emptyset$. We say that $X$ is $Y$-adically complete if the partially-defined left adjoint to the inclusion Sch $\hookrightarrow$ PreStk is defined on $X_{Y}^{\wedge}$ and the canonical map $X_{Y}^{\wedge} \rightarrow X$ realizes $X$ as the value of this functor. If $Y \hookrightarrow X$ is a closed embedding of affine Noetherian DG schemes then this partially defined left adjoint is defined on $X_{Y}^{\wedge}$ and we call its value the $Y$-adic completion of $X$ or the adic completion of $X$ along $Y$.

Remark 1.4. The difference between $X_{Y}^{\wedge}$ and the $Y$-adic completion of $X$ is the same as the usual difference between Spf and Spec. I.e., there is a canonical map $X_{Y}^{\wedge}$ to the $Y$-adic completion in PreStk (but no inverse map). We emphasize that the adic completion is "larger" than the formal completion: for example, if $\operatorname{dim} X>\operatorname{dim} Y$ then the adic completion has generic points which lie outside of $Y$, unlike the formal completion.

We follow [1] in saying an almost finite type scheme $X$ is quasi-smooth if its cotangent complex is perfect of Tor-amplitude $[-1,0]$, and more generally we say that $f: X \rightarrow Y$ is quasi-smooth if $f$ is almost of finite type and the relative tangent complex is perfect of Tor-amplitude $[-1,0]$. For $X$ classical, this coincides with the usual 
condition that $X$ is locally a complete intersection. Recall that quasi-smooth morphisms are Gorenstein (see Section 1.6 for the definition of Gorenstein morphism). We say that $X$ is smooth if its tangent complex is a vector bundle, i.e., perfect of Tor-amplitude 0 ; in this case, $X$ is classical and smooth in the classical sense (see [7] Theorem 9.1.6 or [14] Proposition 8.23).

Example 1.5. If $X, Y$ and $Z$ are smooth schemes and $f: X \rightarrow Z$ and $g: Y \rightarrow Z$ are maps of schemes, then $X \times_{Z} Y$ is a quasi-smooth scheme. When one of $f$ or $g$ is flat, we obtain the standard example of a quasi-smooth classical scheme (i.e., locally complete intersection scheme).

If $f$ is non-flat, the the fiber product is a quasi-smooth scheme but not necessarily classical. For example, $0 \times_{\mathbb{A}^{n}} 0$ (which can be realized as the spectrum of a Koszul complex) is a typical example of a non-classical quasi-smooth scheme.

1.6. Let us recall the format of ind-coherent sheaves from [6].

For any Noetherian scheme $X$, let $\operatorname{Coh}(X)$ denote the full subcategory of $\mathrm{Q} C o h(X)$ consisting of objects which are bounded (i.e., eventually connective and eventually coconnective) and have cohomologies which are finitely generated in $\mathrm{QCoh}\left(X^{\mathrm{cl}}\right)^{\mathrm{P}}$. Let IndCoh $(X)$ denote the DG category obtained as the ind-completion of $\operatorname{Coh}(X)$.

There is a canonical functor $\Psi_{X}: \operatorname{Ind} \operatorname{Coh}(X) \rightarrow \mathrm{Q} \operatorname{Coh}(X)$ obtained as the unique continuous functor inducing the canonical embedding $\operatorname{Coh}(X) \hookrightarrow \mathrm{Q} \operatorname{Coh}(X)$. We denote this functor by $\Psi$ when there is no risk for confusion. If $X$ is regular then $\Psi_{X}$ is an equivalence, but otherwise it is very non-conservative (i.e., it sends many objects to 0$)$. This failure for $\Psi$ to be an equivalence exactly measures the difference between $\operatorname{Coh}(X)$ and $\operatorname{Perf}(X)$ (the DG category of perfect complexes), and therefore $\Psi$ may be understood as measuring the singularities of $X$ in some sense.

IndCoh $(X)$ carries a unique compactly generated $t$-structure such that $\Psi_{X}$ is $t$ exact. In fact, $\Psi_{X}$ induces an equivalence on eventually coconnective objects $\operatorname{Ind} \operatorname{Coh}(X)^{+} \stackrel{\simeq}{\longrightarrow} \mathrm{QCoh}(X)^{+}$.

Remark 1.6. It is a relatively minor technical convenience to work with $\operatorname{Ind} \operatorname{Coh}(X)$ instead of $\operatorname{Coh}(X)$. For example, for a map $f: X \rightarrow Y$ almost of finite type, the functor $f^{!}$is naturally defined as a functor $f^{!}: \operatorname{Ind} \operatorname{Coh}(Y) \rightarrow \operatorname{Ind} \operatorname{Coh}(X)$ and generally does not preserve coherent objects: for $X$ and $Y$ of almost finite type, this condition amounts to being Tor-finite. Working systematically with the ind-completion allows us to ignore such technical conditions by forcing representability of functors and therefore allows for a more uniform treatment of the subject.

For $f: X \rightarrow Y$ a morphism of schemes, recall that $f_{*}: \mathrm{QCoh}(X) \rightarrow \mathrm{Q} \operatorname{Coh}(Y)$ is left $t$-exact and therefore maps eventually coconnective objects to eventually coconnective objects. In particular, $f_{*}(\operatorname{Coh}(X)) \subset \mathrm{Q} \operatorname{Coh}(Y)^{+}$. Therefore, the existence of the $t$ structure on IndCoh as above implies that there exists a unique continuous functor $f_{*}^{\text {IndCoh }}: \operatorname{IndCoh}(X) \rightarrow \operatorname{Ind} \operatorname{Coh}(Y)$ such that $\left.\left(\Psi_{Y} \circ f_{*}^{\text {IndCoh }}\right)\right|_{\operatorname{Coh}(X)}=f_{*} \mid \operatorname{Coh}(X)$ as functors $\operatorname{Coh}(X) \rightarrow \mathrm{Q} \operatorname{Coh}(Y)$. As in [6], $f: X \rightarrow Y$ is eventually coconnective (e.g., Tor-finite or Gorenstein) if and only if $f_{*}^{\text {IndCoh }}$ admits a left adjoint, which we denote $f^{*}$ IndCoh in this case. By definition, $f$ is Gorenstein if and only if $f^{*}$, IndCoh (exists and) differs from $f^{!}$by tensoring with a graded (i.e., cohomologically shifted) line bundle (where $\operatorname{lnd} \operatorname{Coh}(X)$ is regarded as a $\mathrm{QCoh}(X)$-module category in the natural way). Note that [1] Appendix E is a comprehensive reference for Gorenstein morphisms, 
though it contains more precise results than are needed in this text: we only use the definition and the fact that quasi-smooth morphisms are Gorenstein.

Finally, we recall from Section 9 of [6] that IndCoh is defined on any prestack locally almost of finite type.

1.7. We refer to [1] Section 1 for basic facts about quasi-smooth schemes and to loc. cit. Section 2 or [15] Section 2 for Lie algebras in derived algebraic geometry. For the background on ind-schemes we refer to [7] Sections 6 and 7 and for adic completions we refer to [16]. Finally, we refer to [6] Sections 1, 3 and 4 for background on ind-coherent sheaves.

1.8. There is a long tradition of results combining deformation theory and Koszul duality (which we do not pretend to survey here), of which Theorem 1.1 is one example.

We draw the reader's attention in particular to [15] Section 2 where Lurie proves an equivalence between modules over a DG Lie algebra and coherent sheaves on the formal completion of a scheme at a point (this is reviewed in Section 3.2 below). The salient differences between Lurie's theorem and Theorem 1.1 are that the latter concerns the adic completion (rather than the formal completion) and the latter assumes quasi-smoothness.

For our purposes, "Koszul duality" refers merely to the fact that for a compactly generated DG category $\mathcal{C}$ with compact generator $X \in \mathcal{C}$, the canonical functor $\mathcal{C} \rightarrow$ $\operatorname{End}_{\mathcal{C}}(X)^{o p}$-mod given as $Y \mapsto \underline{\operatorname{Hom}}_{\mathcal{C}}(X, Y)$ is an equivalence.

Note that Lurie's theorem is an instance of Koszul duality: see Remark 3.1 below.

Remark 1.7. For a prototypical example of the use of Koszul duality to obtain derived equivalences, which explains the name, see e.g. [3].

1.9. This note is structured as follows. Section 2 contains the formulation and proof of the main result, Theorem 2.1. Section 3 explains how to deduce the above description of coherent sheaves on the formal completion of a quasi-smooth DG scheme. The appendix computes the graded Lie algebra associated to a complete intersection via the Hessian construction.

\section{Coherent sheaves}

2.1. Let $X$ be an affine Noetherian scheme and let $i: Y \rightarrow X$ be a quasi-smooth closed embedding such that $X$ is $Y$-adically complete and $Y$ is almost finite type over $k$. Let $X_{Y}^{\wedge}$ denote the formal completion of $X$ along $Y$, which by Section [7] Section 6 is a prestack locally almost of finite type.

We define the "renormalized" form IndCoh $\operatorname{ren}\left(X_{Y}^{\wedge}\right)$ of $\operatorname{IndCoh}\left(X_{Y}^{\wedge}\right)$ to be the indcompletion of the full subcategory of IndCoh $\left(X_{Y}^{\wedge}\right)$ consisting of objects $\mathfrak{F}$ where $i^{!}(\mathfrak{F}) \in$ $\operatorname{Coh}(Y)$.

Our main result is the following:

Theorem 2.1. There is a unique equivalence of categories:

$$
\operatorname{Ind} \operatorname{Coh}(X) \stackrel{\simeq}{\longrightarrow} \operatorname{Ind} \operatorname{Coh}_{\mathrm{ren}}\left(X_{Y}^{\wedge}\right)
$$

compatible with the canonical functors to $\operatorname{lnd} \operatorname{Coh}\left(X_{Y}^{\wedge}\right)$. 
Remark 2.2. The requirement that $i$ be quasi-smooth is not so restrictive in derived algebraic geometry as in classical algebraic geometry. Indeed, because $X$ is affine $Y$ can be infinitesimally thickened so that $i$ is quasi-smooth and then the above theorem can be applied (the definition of the subcategory at which we renormalize having been modified by this procedure). In fact, in Section 3 we will apply exactly this procedure to the embedding of the closed point of an adically complete quasi-smooth singularity to deduce Theorem 1.1.

2.2. Consider the following condition for an object $\mathfrak{F} \in \operatorname{Ind} \operatorname{Coh}(X)$ to satisfy:

$(*): \mathfrak{F}$ is in the right orthogonal to the subcategory $\operatorname{lnd} \operatorname{Coh}(X \backslash Y) \subset \operatorname{lnd} \operatorname{Coh}(X)$ and $i^{!}(\mathfrak{F})$ is coherent.

The main technical result in the proof of Theorem 2.1 is the following coherence criterion:

Proposition 2.3. $\mathfrak{F}$ is in $\operatorname{Coh}(X)$ if and only if $\mathfrak{F}$ satisfies $(*)$.

The proof of Proposition 2.3 will occupy Sections 2.3-2.12.

Proof that Proposition 2.3 $\Rightarrow$ Theorem 2.1. By [7] Section 7, the natural functor $\operatorname{Ind} \operatorname{Coh}(X) \rightarrow \operatorname{Ind} \operatorname{Coh}\left(X_{Y}^{\wedge}\right)$ is an equivalence when restricted to the right orthogonal to IndCoh $(X \backslash Y)$. Therefore, Proposition 2.3 implies that it induces an equivalence between $\operatorname{Coh}(X)$ and the compact objects in $\operatorname{IndCoh}_{\text {ren }}\left(X_{Y}^{\wedge}\right)$ as desired.

2.3. We begin by showing that if $\mathfrak{F} \in \operatorname{Coh}(X)$ then it satisfies $(*)$.

Because $i$ is Gorenstein, $i^{!}$differs from $i^{* \text {, IndCoh }}$ by tensoring with a graded line bundle. Therefore, we immediately see the for $\mathfrak{F} \in \operatorname{Coh}(X), i^{!}(\mathfrak{F}) \in \operatorname{Coh}(Y)$.

Now the result follows from the following lemma:

Lemma 2.4. If $\mathfrak{F} \in \operatorname{Coh}(X)$, then $\mathfrak{F}$ is in the right orthogonal to $\operatorname{Ind} \operatorname{Coh}(X \backslash Y)$.

Proof. We immediately reduce to the case where $\mathfrak{F} \in \operatorname{Coh}(X)^{\ominus}$. In this case, it is a classical result that coherence for $\mathfrak{F}$ implies that $\mathfrak{F}$ is classically $Y$-adically complete, so in particular a countable filtered limit in $\mathrm{Q} \operatorname{Coh}(Y)^{\odot}$ with surjective structure maps of finitely generated modules set-theoretically supported on $Y$.

Because $X$ is affine, the heart of the $t$-structure is closed under products. Therefore, any countable limit in IndCoh $(X)$ (or QCoh $(X)$ ) consisting of objects in the heart sits in cohomological degrees $[0,1]$. Because the structure maps in the limit above are surjective and because the limit is countable, the limit in $\operatorname{Ind} \operatorname{Coh}(X) \operatorname{or} \mathrm{Q} \operatorname{Coh}(X))$ sits in the heart and coincides with the limit as formed in the heart, i.e., $\mathfrak{F}$ is a (homotopy) limit of objects set-theoretically supported on $Y$. This immediately gives the result.

2.4. It remains to show that if $\mathfrak{F} \in \operatorname{Ind} \operatorname{Coh}(X)$ satisfies $(*)$, then $\mathfrak{F} \in \operatorname{Coh}(X)$. This will be done in Sections 2.5-2.12.

2.5. We begin with the following.

Lemma 2.5. Suppose $\mathfrak{F} \in \operatorname{Ind} \operatorname{Coh}(X)$ is in the right orthogonal to $\operatorname{IndCoh}(X \backslash Y)$ and $i^{!}(\mathfrak{F})$ is in cohomological degrees $\geq 0$. Then $\mathfrak{F}$ is in cohomological degrees $\geq-1$. 
Proof. Suppose $\mathfrak{F}^{\prime} \in \operatorname{Ind} \operatorname{Coh}(X)$ is in cohomological degrees $<-1$. It suffices to show $\operatorname{Hom}\left(\mathfrak{F}^{\prime}, \mathfrak{F}\right)=0$. Let $j$ denote the open embedding $X \backslash Y \hookrightarrow X$. Since $\operatorname{Hom}\left(j_{*}^{\text {IndCoh }}\right.$ $\left.j^{*, \operatorname{lnd} \operatorname{Coh}}\left(\mathfrak{F}^{\prime}\right), \mathfrak{F}\right)=0$, it suffices to show that

$$
\operatorname{Hom}\left(\operatorname{Cone}\left(\mathfrak{F} \rightarrow j_{*}^{\operatorname{lnd} C o h} j^{*, \operatorname{lnd} C o h}\left(\mathfrak{F}^{\prime}\right)\right)[-1], \mathfrak{F}\right)=0 .
$$

$\operatorname{Because} \operatorname{Cone}\left(\mathfrak{F} \rightarrow j_{*}^{\text {IndCoh }} j^{* \text { IndCoh }}\left(\mathfrak{F}^{\prime}\right)\right)$ is in the left orthogonal to $\operatorname{lnd} \operatorname{Coh}(X \backslash Y)$ it suffices to assume $\mathfrak{F}^{\prime}$ itself lies in this left orthogonal and sits in cohomological degrees $<0$.

By [7] Proposition 7.4.5, this left orthogonal is compactly generated by objects of the form $i_{*}^{\text {IndCoh }}(\mathcal{G})$ for $\mathcal{G} \in \operatorname{Coh}(Y)$. Because the $t$-structure is compatible with filtered colimits and compact objects are preserved under truncations, the intersection between this left orthogonal and $\operatorname{Ind} \operatorname{Coh}(X)^{<0}$ is compactly generated by objects of the form $i_{*}^{\text {IndCoh }}(\mathcal{G})$, where $\mathcal{G} \in \operatorname{Coh}^{<0}(Y)$. Therefore it suffices to show:

$$
\operatorname{Hom}\left(i_{*}^{\operatorname{lndCoh}}(\mathcal{G}), \mathfrak{F}\right)=0
$$

for $\mathcal{G} \in \operatorname{Coh}(Y)^{<0}$. But this is clear since we assume that $i^{!}(\mathfrak{F}) \in \operatorname{Ind} \operatorname{Coh}(Y)^{\geq 0}$ and $i_{*}^{\text {IndCoh }}$ is $t$-exact.

Corollary 2.6. Suppose $\mathfrak{F} \in \operatorname{Ind} \operatorname{Coh}(X)$ and $i^{!}(\mathfrak{F})$ is eventually coconnective in IndCoh $(Y)$. Then $\mathfrak{F}$ is eventually coconnective in $\operatorname{lnd} \operatorname{Coh}(X)$.

2.6. Let $\mathfrak{X}$ be a prestack locally almost of finite type. We say $\mathcal{G} \in \operatorname{IndCoh}(\mathfrak{X})$ is eventually connective with coherent cohomologies if for any $S$ a DG scheme almost of finite type over $k$ and for any $\varphi: S \rightarrow \mathfrak{X}$ the object $\varphi^{*}(\mathcal{G}) \in \mathrm{Q} \operatorname{Coh}(S)$ is eventually connective with coherent cohomologies. Let $\mathrm{QCoh}_{\text {coh }}(\mathfrak{X})^{-}$denote the corresponding (non-cocomplete) DG category.

2.7. Let us recall the following result, which is a special case of [16] Theorem 5.3.2.

Theorem 2.7. The canonical functor:

$$
\mathrm{Q} \operatorname{Coh}(X) \rightarrow \mathrm{Q} \operatorname{Coh}\left(X_{Y}^{\wedge}\right)
$$

induces an equivalence:

$$
\mathrm{QCoh}_{\mathrm{coh}}(X)^{-} \rightarrow \mathrm{QCoh}_{\mathrm{coh}}\left(X_{Y}^{\wedge}\right)^{-}
$$

between eventually connective objects with coherent cohomologies.

Now suppose we have a sequence:

$$
Y=Y_{0} \stackrel{\iota_{0}}{\longrightarrow} Y_{1} \stackrel{\iota^{\iota_{1}}}{\longrightarrow} Y_{2} \stackrel{\iota_{2}}{\longrightarrow} \cdots
$$

is a sequence of almost finite type closed subschemes of $X$ such that $X_{Y}^{\wedge}=\operatorname{colim}_{n} Y_{n}$ in PreStk. Let $i_{n}$ denote the closed embedding $Y_{n} \hookrightarrow X$ and let ' ${ }^{\prime} i_{n}$ denote the canonical $\operatorname{map} Y_{n} \rightarrow X_{Y}^{\wedge}$.

In this case, we have the (tautological) equivalence:

$$
\mathrm{Q} \operatorname{Coh}\left(X_{Y}^{\wedge}\right)=\lim _{n}\left(\mathrm{Q} \operatorname{Coh}\left(Y_{n}\right)\right)
$$

the structure maps on the are the functors $\iota_{n}^{*}$ and where the equivalence sends $\mathfrak{F} \in$ $\mathrm{QCoh}\left(X_{Y}^{\wedge}\right)$ to the compatible system $\left\{\mathfrak{F}_{n}\right\}_{n}$ where $\mathfrak{F}_{n}:={ }^{\prime} i_{n}{ }^{*}(\mathfrak{F})$. 
With this notation, we immediately see that the right adjoint to the canonical functor:

$$
\mathrm{Q} \operatorname{Coh}(X) \rightarrow \mathrm{Q} \operatorname{Coh}\left(X_{Y}^{\wedge}\right) \simeq \lim _{n}\left(\mathrm{Q} \operatorname{Coh}\left(Y_{n}\right)\right)
$$

sends a compatible system $\left\{\mathfrak{F}_{n}\right\}_{n}$ to the limit:

$$
\lim _{n} i_{n, *}\left(\mathfrak{F}_{n}\right)
$$

(where the structure maps arise via adjunction from the identification of the *restriction $\mathfrak{F}_{n+k}$ to $Y_{n}$ with $\mathfrak{F}_{n}$ ).

In particular, Theorem 2.7 gives an equivalence given by the same formula between the category $\lim _{n} \mathrm{Q} \operatorname{Coh}_{\mathrm{coh}}\left(Y_{n}\right)^{-}$and $\mathrm{QCoh}$ coh $(X)^{-}$where the notation means "eventually connective objects with coherent cohomologies."

2.8. Now let us return to the proof of Proposition 2.3. Let us fix $\mathfrak{F} \in \operatorname{Ind} \operatorname{Coh}(X)$ satisfying $(*)$.

We claim that there exists $\left\{Y_{n}\right\}$ a system of almost finite type closed subschemes of $X$ as in Section 2.7 such that $X_{Y}^{\wedge}=\operatorname{colim}_{n} Y_{n}$ in PreStk and such that $i_{n}^{*}(\Psi(\mathfrak{F}))$ is eventually connective with coherent cohomologies (as before, $i_{n}$ denotes the embed$\left.\operatorname{ding} Y_{n} \hookrightarrow X\right)$.

This will be treated in Sections 2.9-2.11.

2.9. First, assume that $i: Y \rightarrow X$ can be presented as a global complete intersection. That is, we assume that we have a Cartesian square:

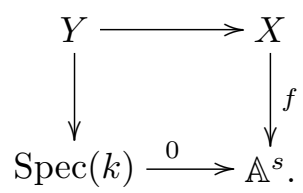

Then we claim that $\left\{Y_{n}\right\}$ can be chosen so that $Y \hookrightarrow Y_{n}$ is a composition of squarezero extensions.

Indeed, define:

$$
Y_{n}:=X \underset{\mathbb{A}^{s}}{\times} \operatorname{Spec}\left(k\left[X_{1}, \ldots, X_{s}\right] /\left(X_{1}^{n}, X_{2}^{n}, \ldots, X_{s}^{n}\right)\right) .
$$

By [7] Section 6, we have $X_{Y}^{\wedge}=\operatorname{colim} Y_{n}$ as desired, and clearly $Y \hookrightarrow Y_{n}$ is a composition of square-zero extensions.

2.10. We continue to assume that $Y \hookrightarrow X$ is a global complete intersection and we let $Y_{n}$ be as in Section 2.9. Then it remains to show that $i_{n}^{*}(\Psi(\mathfrak{F}))$ is eventually connective with coherent cohomologies.

Let us fix the integer $n$ for this section. Let $\mathcal{G}:=i_{n}^{*}(\Psi(\mathfrak{F}))$ and let $\iota$ denote the embedding of $Y$ into $Y_{n}$.

First, note that $\iota^{*}(\mathcal{G}) \in \operatorname{Coh}(Y)$. Indeed, this coincides with $i^{*}(\Psi(\mathfrak{F}))=\Psi\left(i^{\text {IndCoh, } *}\right.$ $(\mathfrak{F})$ ) which lies in Coh $(Y)$ because $i$ is Gorenstein (so $i^{!}$differs from $i^{\text {IndCoh,* }}$ by tensoring with a graded line bundle) and by assumption on $\mathfrak{F}$.

Now the claim that $\mathcal{G} \in \operatorname{Coh}\left(Y_{n}\right)$ follows from the following lemma applied to $\iota: Y \hookrightarrow Y_{n}$ and $\mathcal{G}$. 
Lemma 2.8. Let $\iota: S \hookrightarrow T$ be a closed embedding of Noetherian schemes which is a composition of square-zero extensions. If $\mathcal{G} \in \mathrm{QCoh}(T)$ satisfies $\iota^{*}(\mathcal{G}) \in \mathrm{QCoh}_{\mathrm{coh}}(S)^{-}$ then $\mathcal{G} \in \mathrm{QCoh}_{\mathrm{coh}}(T)^{-}$.

Proof. We immediately reduce to the case where $\iota$ is a square-zero extension. In this case, we have the exact triangle:

$$
\iota_{*}(I) \rightarrow \mathcal{O}_{T} \rightarrow \iota_{*}\left(\mathcal{O}_{S}\right) \stackrel{+1}{\rightarrow}
$$

where $I$ is a connective $\mathcal{O}_{S}$-module with coherent cohomologies. This gives the exact triangle:

$$
\iota_{*}(I) \underset{\mathcal{O}_{T}}{\otimes} \mathcal{G}=\iota_{*}\left(I \underset{\mathcal{O}_{S}}{\otimes} \iota^{*}(\mathcal{G})\right) \rightarrow \mathcal{G} \rightarrow \iota_{*}\left(\mathcal{O}_{S}\right) \underset{\mathcal{O}_{T}}{\otimes} \mathcal{G}=\iota_{*}\left(\iota^{*}(\mathcal{G})\right) \stackrel{+1}{\rightarrow}
$$

immediately implying the result.

2.11. Now let us complete the proof of the claim from Section 2.8 in the general case, i.e., when $i$ is not assumed to be a global complete intersection.

First, recall from [7] Section 6 that there exists some $\left\{Y_{n}\right\}$ with $\operatorname{colim}_{n} Y_{n}=X_{Y}^{\wedge}$. In fact, we claim that for any such choice of $Y_{n}$ we have the desired property, i.e., satisfy $i_{n}^{*}(\Psi(\mathfrak{F})) \in \mathrm{QCoh}_{\mathrm{coh}}\left(Y_{n}\right)^{-}$.

Indeed, note that this statement is étale local on $X$. Therefore, we can assume that $i$ is given as a complete intersection. Then, as above, we can choose a sequence $\left\{Y_{m}^{\prime}\right\}$ such that for each $i_{m}^{\prime}: Y_{m} \hookrightarrow X$ we have $i_{m}^{* *}(\Psi(\mathfrak{F})) \in \mathrm{QCoh}_{\mathrm{coh}}\left(Y_{m}\right)^{-}$and $\operatorname{colim}_{m} Y_{m}^{\prime}=X_{Y}^{\wedge}$.

But since the map $i_{n}: Y_{n} \hookrightarrow X$ factors through $X_{Y}^{\wedge}$, it must factor through some $Y_{m}^{\prime}$. Therefore, $i_{n}^{*}(\Psi(\mathfrak{F}))$ is obtained by $*$-restriction from some $i_{m}^{\prime *}(\Psi(\mathfrak{F}))$ and since this lies in QCoh ${ }_{\mathrm{coh}}\left(Y_{m}\right)^{-}$we obtain the desired result.

2.12. Now we deduce Proposition 2.3. Let $\left\{Y_{n}\right\}$ be as in Section 2.8 .

Suppose $\mathfrak{F} \in \operatorname{Ind} \operatorname{Coh}(X)$ satisfies $(*)$. Because $\mathfrak{F}$ is eventually coconnective (by Corollary 2.6) and lies in the right orthogonal to $\operatorname{lnd} \operatorname{Coh}(X \backslash Y)$, we see that $\Psi(\mathfrak{F})$ is also eventually coconnective and lies in the right orthogonal to $\mathrm{Q} \operatorname{Coh}(X \backslash Y)$. Therefore, $\Psi(\mathfrak{F})$ is $Y$-adically complete, i.e., the morphism:

$$
\Psi(\mathfrak{F}) \rightarrow \lim i_{n, *} i_{n}^{*}(\Psi(\mathfrak{F}))
$$

is an equivalence. By Section 2.8 we have $i_{n}^{*}(\Psi(\mathfrak{F})) \in \mathrm{QCoh}_{\mathrm{coh}}\left(Y_{n}\right)^{-}$. Therefore, by Section 2.7 we see that $\Psi(\mathfrak{F}) \in \mathrm{QCoh}_{\mathrm{coh}}(X)^{-}$. But by Corollary 2.6 we know that $\Psi(\mathfrak{F})$ is eventually coconnective in $\mathrm{Q} C o h(X)$, giving the desired result.

\section{The quasi-smooth case}

3.1. In this section, we deduce Theorem 1.1 from Theorem 2.1. 
3.2. Let us recall some facts about Lie algebras in derived algebraic geometry.

Let $Z$ be a DG scheme almost of finite type over $k$ and let $z \in Z$ be a closed point. Let $\mathfrak{g}_{Z, z}$ be the corresponding Lie algebra. Then by [15] there is an equivalence: $\operatorname{lnd} \operatorname{Coh}\left(Z_{z}^{\wedge}\right) \simeq \mathfrak{g}_{Z, z}-\bmod$ such that the diagram:

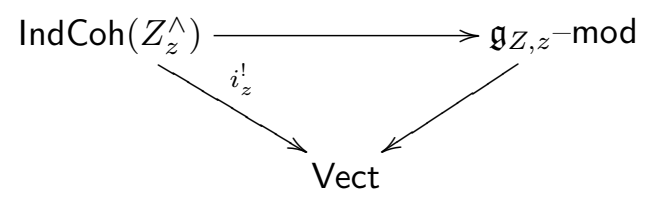

commutes (here $\mathfrak{g}_{Z, z}-\bmod \rightarrow$ Vect is the canonical forgetful functor).

Remark 3.1. Since $i_{z, *}^{\operatorname{Ind} \operatorname{Coh}}(k(z))$ is a compact generator for $\operatorname{IndCoh}\left(Z_{z}^{\wedge}\right)$, this theorem amounts to a calculation of $\operatorname{End}\left(i_{z, *}(k(z))\right)$.

3.3. Suppose $Z$ is a quasi-smooth scheme over $k$ and let $z \in Z$ be a closed point. Let $\widehat{Z}$ denote the adic completion of $Z$ at $z$. As in the introduction, let $\mathfrak{g}_{Z, z}-\bmod _{f . g}$. denote the full subcategory of $\mathfrak{g}_{Z, z}$-mod consisting of objects $M$ such that $H^{*}(M)$ is finitely generated over $H^{*}\left(\mathfrak{g}_{Z, z}\right)$, i.e., over $\operatorname{Sym}\left(H^{2}\left(\mathfrak{g}_{Z, z}\right)\right)$.

The main result of this section is the following:

Theorem 3.2. There is an equivalence of categories:

$$
\operatorname{Ind} \operatorname{Coh}(\widehat{Z}) \simeq \mathfrak{g}_{Z, z}-\bmod _{\mathrm{ren}}:=\operatorname{lnd}\left(\mathfrak{g}_{Z, z}-\bmod _{f . g .}\right)
$$

Remark 3.3. One can deduce similar equivalences for some related categories of sheaves.

More precisely, let $\mathfrak{g}_{Z, z}-\bmod _{\text {f.d. }}$ denote the full subcategory of $\mathfrak{g}_{Z, z}$-mod consisting of modules $M$ such that as a mere vector space $M$ is bounded cohomologically with finite-dimensional cohomologies and let $\mathfrak{g}_{Z, z}-\bmod _{\text {perf }}$ denote the category of $\mathfrak{g}_{Z, z}$-modules compact in $\mathfrak{g}_{Z, z}-\bmod$. Let $\mathfrak{g}_{Z, z}-\bmod _{f . d . / p e r f}$ denote $\mathfrak{g}_{Z, z}-\bmod _{f . d} \cap$ $\mathfrak{g}_{Z, z}-\bmod _{\text {perf }}$.

Then we have equivalences $\mathrm{Q} \operatorname{Coh}(\widehat{Z}) \simeq \operatorname{lnd}\left(\mathfrak{g}_{Z, z}-\bmod _{f . d .}\right)$ and $\mathrm{Q} \operatorname{Coh}\left(Z_{z}^{\wedge}\right) \simeq$ $\operatorname{Ind}\left(\mathfrak{g}_{Z, z}-\bmod _{\text {f.d./perf }}\right)$. Indeed, by Theorem 3.2 the former equivalence amounts to the claim that $\mathfrak{F} \in \operatorname{Coh}(\widehat{Z})$ lies in $\operatorname{Perf}(\widehat{Z})$ if and only if $i_{z}^{!}(\mathfrak{F})$ is bounded with finitedimensional cohomologies. Let $\Xi$ denote the left adjoint to $\Psi$. Then the claim follows because we have:

$$
i_{z}^{!}(\mathfrak{F})=i_{z}^{*}(\Psi(\mathfrak{F})) \otimes i_{z}^{!}\left(\Xi\left(\mathcal{O}_{\widehat{Z}}\right)\right)
$$

and $i_{z}^{!}\left(\Xi\left(\mathcal{O}_{\widehat{Z}}\right)\right)$ is a shifted line because $\widehat{Z}$ is Gorenstein. This equivalence can also been seen directly from Lurie's theorem describing $\mathfrak{g}_{Z, z}-\bmod$ (i.e., without appeal to Theorem 2.1) by using the picture from [7] Section 7 realizing $\operatorname{Ind} \operatorname{Coh}\left(Z_{z}^{\wedge}\right)$ as ind-coherent sheaves on $Z$ set-theoretically supported at $z$, or less directly (but fundamentally by the same argument) by using the theory of singular support as developed in [1]. 
These equivalences identify the diagrams:

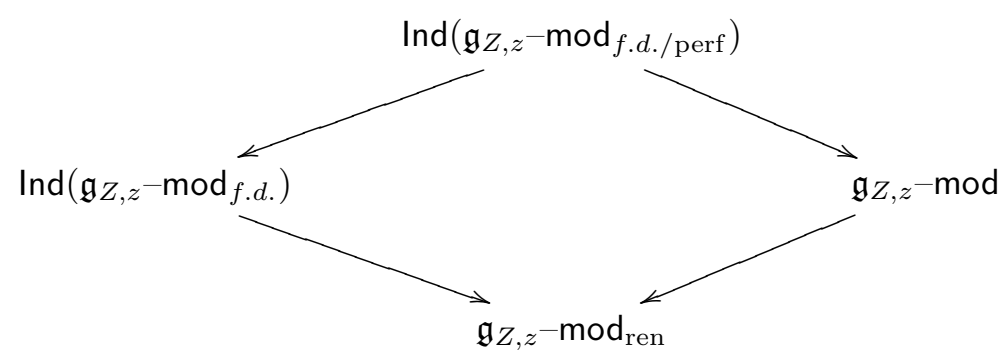

and

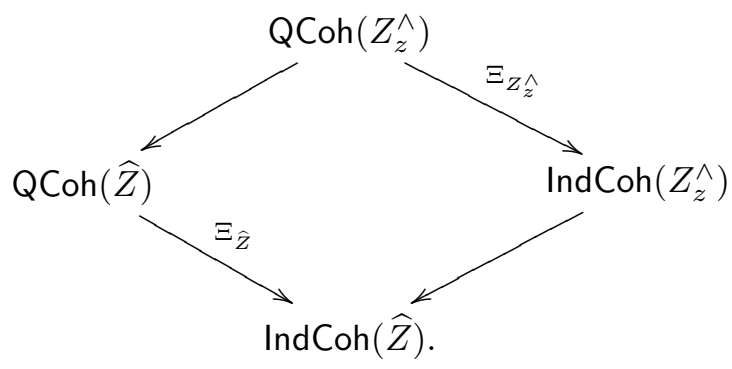

Here $\mathrm{Q} \operatorname{Coh}\left(Z_{z}^{\wedge}\right) \rightarrow \mathrm{Q} \operatorname{Coh}(\widehat{Z})$ is the left adjoint to the canonical *-restriction functor and $\operatorname{Ind} \operatorname{Coh}\left(Z_{z}^{\wedge}\right) \rightarrow \operatorname{Ind} \operatorname{Coh}(\widehat{Z})$ is the left adjoint to the canonical !-restriction functor.

3.4. We will need the following result:

Lemma 3.4. Suppose $A$ is a commutative algebra (without any co/connectivity assumptions). Suppose that $H^{*}(A)$ is concentrated in even degrees (i.e., A has no non-vanishing odd cohomology groups) and that the commutative algebra $H^{*}(A)=$ $\oplus_{n \in \mathbb{Z}} H^{2 n}(A)$ is a regular (in particular, Noetherian) ring. Finally, suppose that every graded-projective module over $H^{*}(A)$ is graded-free. Then an object $M \in A$-mod is perfect if and only if $H^{*}(M)$ is finitely generated over $H^{*}(A)$.

Remark 3.5. In the setting of a (non-super) commutative $\mathbb{Z}$-graded ring $B=\oplus B_{i}$ it is not so terribly uncommon for every finitely generated graded-projective module to be graded-free. E.g., it holds if $B$ is Noetherian, $B_{i}=0$ for $i<0$ (or $i>0$ ) and $B_{0}$ is a field.

Proof of Lemma 3.4. First, let us show that if $M$ is perfect, then $H^{*}(M)$ is finitely generated over $H^{*}(A)$. Clearly the result holds for $M=A$ and is preserved under direct summands. Therefore, it suffices to show that if $f: M \rightarrow M^{\prime}$ is a morphism where $H^{*}(M)$ and $H^{*}\left(M^{\prime}\right)$ are finitely generated over $H^{*}(A)$, then $H^{*}(\operatorname{Cone}(f))$ is finitely generated as well. We have a short exact sequence:

$$
0 \rightarrow \operatorname{Coker}\left(H^{*}(f)\right) \rightarrow H^{*}(\operatorname{Cone}(f)) \rightarrow \operatorname{Ker}\left(H^{*+1}(f)\right) \rightarrow 0
$$

and therefore the result follows immediately from the Noetherian assumption.

Now suppose that $H^{*}(M)$ is finitely generated over $H^{*}(A)$ : we need to show that $M$ is perfect. We will proceed by descending induction on the homological dimension of $H^{*}(M)$ in the category of graded $H^{*}(A)$-modules (recall that if $B$ is a graded Noetherian ring which is regular as a (non-graded) ring then its category of graded 
modules has finite homological dimension as well). Because $H^{*}(M)$ is a finitely generated graded module, we can choose a map $\varepsilon: \oplus_{i=1}^{n} A\left[r_{i}\right] \rightarrow M$ inducing a surjection $\oplus_{i=1}^{n} H^{*+r_{i}}(A) \rightarrow H^{*}(M)$. Then we claim that the homological dimension of $H^{*}(\mathrm{Cone}(\varepsilon))$ as an $H^{*}(A)$-module is strictly less than the homological dimension of $H^{*}(M)$. Indeed, we have the short exact sequence:

$$
0 \rightarrow H^{*}(\text { Cone }(\varepsilon)) \rightarrow \oplus_{i=1}^{n} H^{*+r_{i}}(A) \rightarrow H^{*}(M) \rightarrow 0 .
$$

By induction, this reduces us to the case of homological dimension zero.

In this case, by assumption the module $H^{*}(M)$ is graded-free. As above, we choose a basis of $H^{*}(M)$ to obtain $\oplus_{i=1}^{n} A\left[r_{i}\right] \rightarrow M$ inducing an isomorphism at the level of cohomology, i.e., such that the map is an equivalence.

3.5. Now let us prove Theorem 3.2.

The problem is Zariski local on $Z$ and therefore we may assume $Z$ is given as a global complete intersection with $U$ and $V$ smooth:

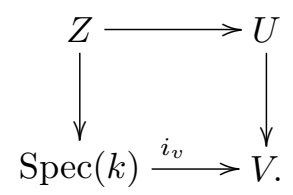

This defines the quasi-smooth closed embedding:

$$
\varphi: \mathcal{G}:=\operatorname{Spec}(k) \underset{V}{\times} \operatorname{Spec}(k) \rightarrow Z .
$$

By Theorem 2.1 and Section 3.2, it suffices to show $\mathfrak{F} \in \operatorname{Ind} \operatorname{Coh}\left(Z_{z}^{\wedge}\right)$ satisfies $\varphi^{!}(\mathfrak{F}) \in \operatorname{Coh}(\mathcal{G})$ if and only if $H^{*}\left(i_{z}^{!}(\mathfrak{F})\right)$ is finitely generated over $H^{*}\left(\mathfrak{g}_{Z, z}\right)$.

By Section 3.2, we have the equivalences

$$
\begin{gathered}
\operatorname{Ind} \operatorname{Coh}\left(Z_{z}^{\wedge}\right) \stackrel{\simeq}{\longrightarrow} \mathfrak{g}_{Z, z}-\bmod , \\
\operatorname{Ind} \operatorname{Coh}\left(\mathcal{G}_{z}^{\wedge}\right)=\operatorname{Ind} \operatorname{Coh}(\mathcal{G}) \stackrel{\simeq}{\longrightarrow} \mathfrak{g}_{\mathcal{G}, z}-\bmod =\operatorname{Sym}\left(T_{V, v}[-2]\right)-\bmod ,
\end{gathered}
$$

so that the functor $\varphi^{!}$corresponds to the restriction along the canonical map $T_{V, v}[-2]$ $\rightarrow \mathfrak{g}_{Z, z}$. Therefore, we see that $\varphi^{!}(\mathfrak{F}) \in \operatorname{Coh}(\mathcal{G})$ if and only if $i_{z}^{!}(\mathfrak{F})$ is perfect in $T_{V, v}[-2]-$ mod. By Lemma 3.4, this is equivalent to asking that $H^{*}\left(i_{z}^{!}(\mathfrak{F})\right)$ be finitely generated over $H^{*}\left(\operatorname{Sym}\left(T_{\mathcal{G}, z}[-2]\right)\right)\left(=\operatorname{Sym}\left(T_{V, v}\right)\right.$ where generators sit in degree 2$)$.

But since $H^{1}\left(T_{\mathcal{G}, z}\right)=T_{V, v} \rightarrow H^{1}\left(T_{Z, z}\right)$ is a surjection, we see that this condition is equivalent to asking that $H^{*}\left(i_{z}^{!}(\mathfrak{F})\right)$ is finitely generated over $\operatorname{Sym}\left(H^{1}\left(T_{Z, z}\right)\right)$, as desired.

\section{Acknowledgments}

It is a pleasure to thank Dennis Gaitsgory and Sasha Beilinson for many conversations and suggestions about this note and for their encouragement to publish it.

\section{Appendix A. Hessian calculations}

A.1. In this appendix, we assume a good formalism of Lie algebroids in derived algebraic geometry. Such a theory has not yet been written down but will appear in Gaitsgory-Rozenblyum [8]. We will state the principal constructions we need from the theory in Section A.6. 
A.2. Let $f: X \rightarrow Y$ be a map between smooth schemes and let $T_{X / Y} \in \operatorname{Perf}(X)$ be its tangent complex. This is a Lie algebroid on $X$, so in particular $T_{X / Y}[-1]$ is equipped with a natural structure of Lie algebra in $\mathrm{QCoh}(X)$. Indeed, this follows from the formal groupoid picture for Lie algebroids by an analysis as in [1] Section 2.

Remark A.1. This homotopy Lie algebra structure on the shifted tangent complex is classical. The idea dates back to the classical work [9-11] of Grothendieck-Illusie on the cotangent complex. It seems to be given at the cochain level first in [18], which also gives references to previous work at the associated graded level. This homotopy Lie algebra structure was also presented in [4] in a more conceptual framework. In the smooth case, it was described differential geometrically in [12], emphasizing the role of Atiyah classes [2].

In the framework of higher category theory, this construction is cleaner: see [5], $[1,15]$ for complementary viewpoints.

We thank the referee for help with giving the proper citations for this structure.

In this appendix, we will explain how to to explicitly compute the corresponding graded Lie algebra (in the "super" sense) $H^{*}\left(T_{X / Y}[-1]\right)$ in $\mathrm{Q} \operatorname{Coh}(X)^{\varnothing}$.

More generally, suppose $S$ is a classical scheme and $x \in X(S)$. Then the symmetric monoidal functor $x^{*}: \mathrm{Q} \operatorname{Coh}(X) \rightarrow \mathrm{Q} \operatorname{Coh}(S)$ gives a Lie algebra structure on $x^{*}\left(T_{X / Y}[-1]\right)$ in $\mathrm{Q} \operatorname{Coh}(S)$. We will compute the corresponding graded Lie algebra $H^{*}\left(x^{*}\left(T_{X / Y}[-1]\right)\right)$ in $\mathrm{QCoh}(S)^{\odot}$.

This graded Lie algebra has non-zero graded components only in degrees 1 and 2 (because $S$ was assumed classical) and therefore its bracket is encoded entirely by a map:

$$
\operatorname{Sym}^{2}\left(H^{0}\left(x^{*}\left(T_{X / Y}\right)\right)\right) \rightarrow H^{1}\left(x^{*}\left(T_{X / Y}\right)\right)
$$

in $\mathrm{Q} \operatorname{Coh}(S)^{\mathrm{P}}$.

A.3. Let $\left.d f\right|_{x}$ denote the map $x^{*}\left(T_{X}\right) \rightarrow x^{*} f^{*}\left(T_{Y}\right)$ obtained by applying $x^{*}$ to the differential of $f$.

Recall that in addition to $\left.d f\right|_{x}$, we have the classical Hessian map (alias: second derivative):

$$
\begin{aligned}
\operatorname{Hess}_{x}(f): \operatorname{Sym}^{2}\left(H^{0}\left(x^{*}\left(T_{X / Y}\right)\right)\right) & =\operatorname{Sym}^{2}\left(\operatorname{Ker}\left(\left.d f\right|_{x}\right)\right) \rightarrow \operatorname{Coker}\left(\left.d f\right|_{x}\right) \\
& =H^{1}\left(x^{*}\left(T_{X / Y}\right)\right) .
\end{aligned}
$$

(We will review the construction of the Hessian in Section A.5.)

We will show the following:

Proposition A.2. The Lie bracket $\operatorname{Sym}^{2}\left(H^{0}\left(x^{*}\left(T_{X / Y}\right)\right)\right) \rightarrow H^{1}\left(x^{*}\left(T_{X / Y}\right)\right)$ coincides with the Hessian.

A.4. Before proving the proposition, we note that this computes "explicitly" the graded Lie algebras associated to the classical points of a global complete intersection.

Indeed, let $y \in Y$ be a closed point and let $Z$ be the derived fiber product of $f$ over $y$. Let $z$ be an $S$-point of $Z$ where $S$ is a classical scheme. We abuse notation by 
denoting the corresponding $S$-point of $X$ also by $z$. Therefore, we have the diagram:

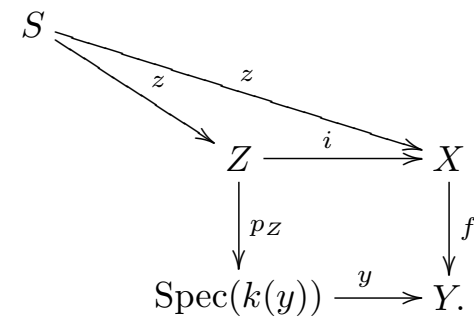

Observe that we have the natural morphisms:

$$
\begin{gathered}
H^{0}\left(z^{*}\left(T_{Z}\right)\right) \stackrel{z^{*}(d i)}{\longrightarrow} H^{0}\left(\operatorname{Ker}\left(\left.d f\right|_{z}\right)\right) \subset H^{0}\left(z^{*}\left(T_{X}\right)\right), \\
H^{0}\left(z^{*} p_{Z}^{*}\left(T_{Y, y}\right)\right)=H^{0}\left(z^{*} f^{*}\left(T_{Y}\right)\right) \rightarrow H^{0}\left(\operatorname{Coker}\left(\left.d f\right|_{z}\right)\right) \rightarrow H^{1}\left(z^{*} f^{*}\left(T_{Z}\right)\right)
\end{gathered}
$$

coming from the exact triangle:

$$
z^{*} T_{Z}=z^{*}\left(T_{X / Y}\right) \longrightarrow z^{*}\left(T_{X}\right) \longrightarrow z^{*}\left(T_{Y}\right)
$$

by taking cohomology.

Composing these maps with $i^{*}(\operatorname{Hess}(f))$, we obtain a map:

$$
\operatorname{Sym}^{2}\left(H^{0}\left(z^{*}\left(T_{Z}\right)\right)\right) \rightarrow H^{1}\left(z^{*}\left(T_{Z}\right)\right) .
$$

By Proposition A.2, this map coincides with the Lie bracket on the graded Lie algebra $H^{*}\left(z^{*}\left(T_{Z}[-1]\right)\right)$ in $\mathrm{Q} \operatorname{Coh}(S)^{\ominus}$.

Indeed, this follows because symmetric monoidal functor $i^{*}$ induces an equivalence of Lie algebras in $\mathrm{QCoh}(Z)$ :

$$
i^{*}\left(T_{X / Y}[-1]\right) \stackrel{\simeq}{\longrightarrow} T_{Z}[-1] .
$$

A.5. Next, we recall the construction of the Hessian $\operatorname{Hess}_{x}(f)$ of the map $f: X \rightarrow Y$ for $x \in X(S)$.

Let $D_{X}$ denote the sheaf of differential operators on $X$ and let $F$. be the usual filtration of $D_{X}$ by order of the differential operator, and similarly for $Y$. Recall that there is the canonical morphism $D_{X} \rightarrow f^{*}\left(D_{Y}\right)$ of filtered $D_{X}$-modules inducing $\operatorname{Sym}^{\bullet}(d f)$ on the associated graded.

We obtain a commutative diagram in $\mathrm{Q} \operatorname{Coh}(X)^{\odot}$ where the rows are exact:

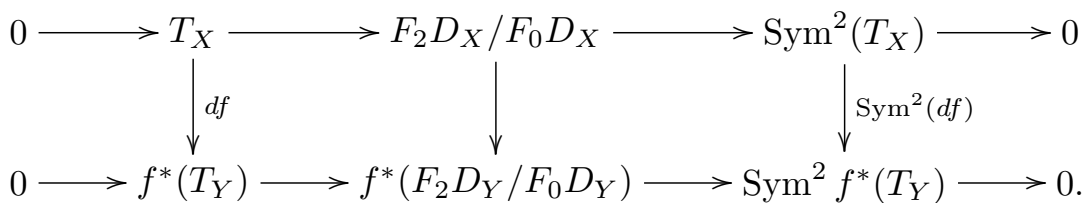

Applying $x^{*}$, we obtain a similar commutative diagram in $\mathrm{Q} \operatorname{Coh}(S)^{\complement}$ :

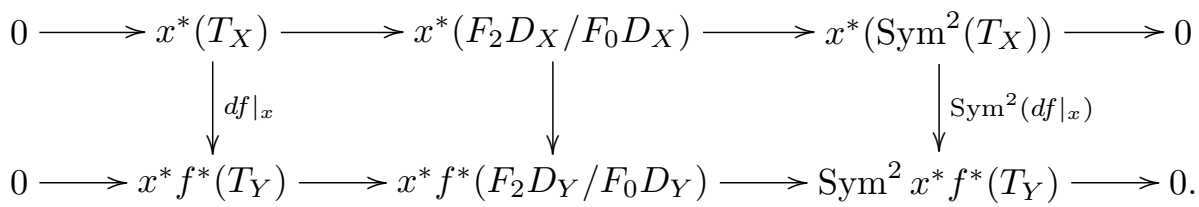


By definition, the Hessian is obtained by composing the boundary morphism:

$$
\operatorname{Ker}\left(\operatorname{Sym}^{2}\left(\left.d f\right|_{x}\right)\right) \rightarrow \operatorname{Coker}\left(\left.d f\right|_{x}\right)
$$

with the tautological map:

$$
\operatorname{Sym}^{2}\left(\operatorname{Ker}\left(\left.d f\right|_{x}\right)\right) \rightarrow \operatorname{Ker}\left(\operatorname{Sym}^{2}\left(\left.d f\right|_{x}\right)\right) .
$$

Remark A.3. Suppose $X=\mathbb{A}^{n}$ and $Y=\mathbb{A}^{m}$ and let $f=\left(f_{1}, \ldots, f_{m}\right)$. Then $\operatorname{Hess}(f)=$ $\operatorname{Hess}_{\mathrm{id}_{X}}(f)$ naturally factors in the obvious way through the map:

$$
\operatorname{Sym}^{2}\left(T_{X}\right)=\operatorname{Sym}^{2}\left(\mathcal{O}_{X}\left[\partial_{1}, \ldots, \partial_{n}\right]\right) \rightarrow f^{*}\left(T_{Y}\right)=\mathcal{O}_{X}\left[\partial_{1}^{\prime}, \ldots, \partial_{m}^{\prime}\right]
$$

defined by the classical formula:

$$
\partial_{i} \cdot \partial_{j} \mapsto \sum_{k=1}^{m} \frac{\partial^{2} f_{j}}{\partial x_{j} \partial x_{i}} \cdot \partial_{j}^{\prime} .
$$

Remark A.4. We note that the Hessian may be also be defined via the dual description of infinitesimal neighborhoods of the diagonal in place of differential operators.

A.6. Proposition A.2 is based on two standard compatibilities, which probably lack a reference presently.

First, suppose that $L$ is a Lie algebra in a "nice" symmetric monoidal DG category $\mathscr{C}$ (here "nice" means admitting all colimits and the tensor product commutes with colimits in each variable). Let $C(L)$ be the (homological) Chevalley complex of $L$ and let $F$. denote the canonical filtration on $C(L)$. Then $\operatorname{Gr}_{i}^{F}(C(L))$ canonically identifies with $\operatorname{Sym}^{i}(L[1])$, so in particular we obtain a canonical map:

$$
\Lambda^{2}(L):=\operatorname{Sym}^{2}(L[1])[-2] \rightarrow L
$$

as from the corresponding filtration of Cone $\left(F_{0} C(L) \rightarrow F_{2} C(L)\right)$. This map is (homotopic to) the Lie bracket for $L$.

Second, let $T$ be a scheme and let $L$ be an algebroid in $\operatorname{Perf}(T) \subset \mathrm{Q} \operatorname{Coh}(T)$. Then $L[-1]$ carries a natural structure of Lie algebra in $\mathrm{Q} C o h(T)$. With respect to this structure, the Chevalley complex $C(L[-1])$ naturally coincides as a filtered coalgebra in QCoh $(T)$ with the enveloping algebroid $U(L)$.

A.7. With these preliminaries, Proposition A.2 follows by the following diagram chase.

Let $U\left(T_{X / Y}\right)$ denote the enveloping algebroid of $T_{X / Y}$, which we regard as a mere filtered object of $\mathrm{Q} C \mathrm{Ch}(X)$. We have a commutative diagram where all maps are compatible with filtrations and $\varepsilon$ is the augmentation:

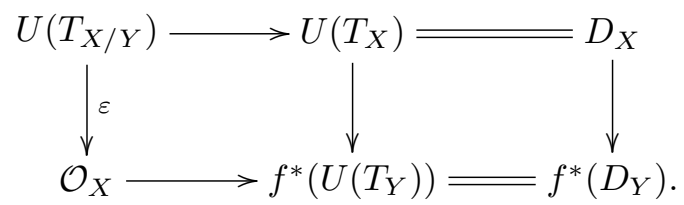

Indeed, it suffices to show that $\mathcal{O}_{X} \rightarrow f^{*}\left(D_{Y}\right)$ is a morphism of $T_{X / Y}$-modules. To see this, note that $\mathcal{O}_{Y} \rightarrow D_{Y}$ is a map of modules for the trivial algebroid on $\mathcal{O}_{Y}$ and that $T_{X / Y}$ is the algebroid pull-back of the trivial algebroid.

In particular, the natural morphism:

$$
\text { Cone }\left(\mathcal{O}_{X}=F_{0} U\left(T_{X / Y}\right) \rightarrow U\left(T_{X / Y}\right)\right) \rightarrow f^{*}\left(D_{Y} / F_{0} D_{Y}\right)
$$


is canonically trivialized and therefore we obtain a natural filtered map:

$$
\text { Cone }\left(F_{0} U\left(T_{X / Y}\right) \rightarrow U\left(T_{X / Y}\right)\right) \rightarrow \text { Cone }\left(D_{X} / F_{0} D_{X} \rightarrow f^{*}\left(D_{Y} / F_{0} D_{Y}\right)\right)[-1] .
$$

Thus, we obtain the commutative diagram between exact triangles:

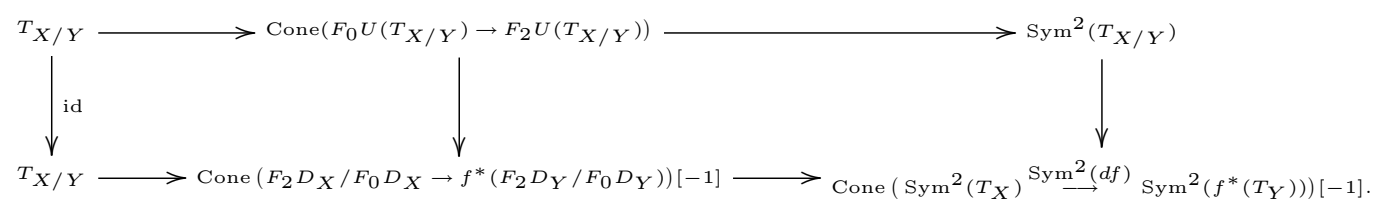

We deduce a natural commutative diagram:

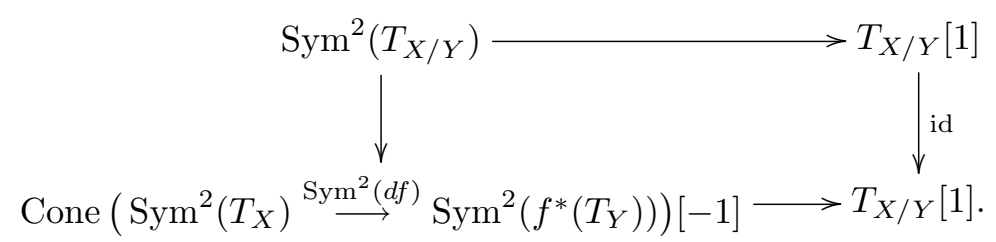

By Section A.6, the composition is equal (i.e., homotopic) to the Lie bracket.

Applying $x^{*}$ and passing to cohomology, we obtain the commutative diagram:

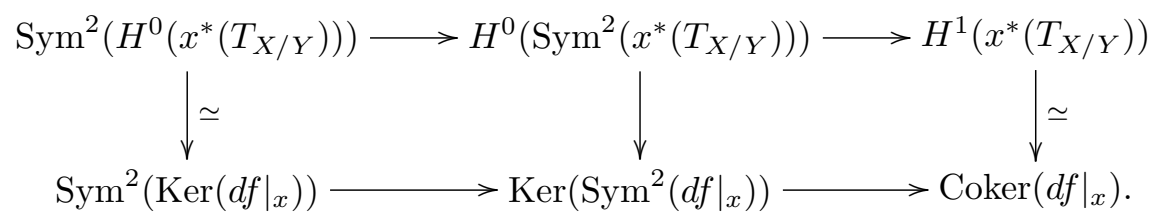

By definition, the composition of the bottom row computes $\operatorname{Hess}_{x}(f)$. Moreover, as above, the composition of the top row computes the Lie bracket at the level of cohomology. This completes the computation.

\section{References}

[1] D. Arinkin and D. Gaitsgory, Singular support of coherent sheaves and the geometric Langlands conjecture, 2012, arXiv:1201.6343.

[2] M. Atiyah, Complex analytic connections in fibre bundles, Trans. Amer. Math. Soc. 85 (1957), 181-207.

[3] A. Beilinson, V. Ginzburg and W. Soergel, Koszul duality patterns in representation theory, J. Amer. Math. Soc. 9(2) (1996), 473-528.

[4] I. Ciocan-Fontanine and M. Kapranov, Derived Quot schemes, Ann. Sci. École Norm. Sup. (4) 34(3) (2001), 403-440.

[5] J. Francis, The tangent complex and Hochschild cohomology of $E_{n}$-rings, available at: http://arxiv.org/abs/1104.0181

[6] D. Gaitsgory, Ind-coherent sheaves, arXiv:1105.4857.

[7] D. Gaitsgory and N. Rozenblyum, DG Indschemes, arXiv:1108.1738.

[8] - Studies in derived algebraic geometry, Forthcoming.

[9] A. Grothendieck, Catégories cofibrées additives et complexe cotangent relatif, Lecture Notes in Mathematics, No. 79, Springer-Verlag, Berlin, 1968.

[10] L. Illusie, Complexe cotangent et déformations, I, Lecture Notes in Math., 239, Springer-Verlag, Berlin, 1971.

[11] - Complexe cotangent et déformations, II, Lecture Notes in Math., 283, Springer-Verlag, Berlin, 1972.

[12] M. Kapranov, Rozansky-Witten invariants via Atiyah classes, Composit. Math. 115(1) (1999), 71-113. 
[13] J. Lurie, Higher topos theory, 170 Annals of Mathematics Studies, Princeton University Press, Princeton, NJ, 2009.

[14] — DAG VII: Spectral schemes, 2011, available at: http://math.harvard.edu/ lurie/papers/DAG-VII.pdf.

[15] — DAG X: Formal moduli problems, 2011, available at http://math.harvard.edu/ lurie/papers/DAG-X.pdf.

[16] — DAG XII: Proper morphisms, completions, and the Grothendieck existence theorem, 2011, available at: http://math.harvard.edu/ lurie/papers/DAG-XII.pdf.

[17] — Higher algebra, 2012, available at: http://math.harvard.edu/ lurie/papers/Higher Algebra.pdf.

[18] M. Schlessinger and J. Stasheff, The Lie algebra structure of tangent cohomology and deformation theory, J. Pure Appl. Algebra 38(2-3) (1985), 313-322.

Harvard University, 1 Oxford Street, Cambridge, MA, USA

E-mail address: sraskin@math.harvard.edu 
